\title{
NEAR-DROWNING EARLY PREDICTION TECHNIQUE USING NOVEL EQUATIONS (NEPTUNE) FOR SWIMMING POOLS
}

\author{
B David Prakash
}

IAG Firemark, Singapore

\begin{abstract}
Safety is a critical aspect in all swimming pools. This paper describes a near-drowning early prediction technique using novel equations (NEPTUNE). NEPTUNE uses equations or rules that would be able to detect near-drowning using at least 1 but not more than 5 seconds of video sequence with no false positives. The backbone of NEPTUNE encompasses a mix of statistical image processing to merge images for a video sequence followed by K-means clustering to extract segments in the merged image and finally a revisit to statistical image processing to derive variables for every segment. These variables would be used by the equations to identify near-drowning. NEPTUNE has the potential to be integrated into a swimming pool camera system that would send an alarm to the lifeguards for early response so that the likelihood of recovery is high.
\end{abstract}

\section{KEYWORDS}

Near-drowning Detection, Drowning Detection, Statistical Image Processing, K-means Clustering, Swimming Pools

\section{INTRODUCTION}

The World Health Organization (WHO) classifies drowning as the 3rd leading cause of unintentional injury worldwide [1]. Globally, the highest drowning rates are among children aged between 1 to 4 years, followed by children aged between 5 to 9 years [1]. In individual countries such as the United States and France, within a short time frame of about 4 months, the number of drowning deaths in swimming pools and spas ranged between 74 and 163 [2-3]. Studies have shown that lifeguards may not be trained well enough to handle a drowning situation [4]. Hence, having a drowning detection system in conjunction with lifeguards in swimming pools would aid to promote swimming pool safety.

The existing drowning detection technologies can be broadly categorized into vision based systems [5-10] and wearable sensor based systems [11-13]. Vision based technologies can be further sub-categorized into those using underwater cameras [5-6] and those employing above water cameras [7-10]. A limitation of the use of underwater cameras is that they might miss the initial struggle that might take place above the water. Some drawbacks of the existing above water camera vision based technologies are that they have been demonstrated only using simulated video [7-10], they are trained to detect above water motionlessness [10] instead of the Dhinaharan Nagamalai et al. (Eds) : CSEIT, NCS, SPM, NeTCoM - 2018 pp. 151-169, 2018. (C) CS \& IT-CSCP 2018

DOI : $10.5121 /$ csit.2018.81812 
struggling motion which might pre-occur or might require additional costly fixtures such as a microarray to be mounted above the water to cover the entire swimming pool [7]. The shortcoming of a wearable based system is primarily the discomfort of use [8] which has an unproven possible notion that it might lead to younger children attempting to eliminate the discomfort by removing the device.

NEPTUNE is aimed at targeting the integration into existing above water camera(s) to enable a cost-effective installation by utilizing images from an existing camera fixture. It can identify predrowning struggling motions early using at least 1 but not more than 5 seconds of video sequence. The detection equations that NEPTUNE uses were derived from video sequences using an actual video footage [14-15] *.

*Please be informed that Fig. 1. contains confronting and real still images of a pre-drowning struggling victim.

\section{NEPTUNE}

\subsection{Dataset}

Two sets of videos were downloaded [14-15]. The first came with a manual red contour segmentation of the drowning victim throughout the entire video [14] and the second had the red contour segmentation of the drowning victim only in the initial portion of the video prior to the start of the pre-drowning struggle [15]. The second video [15] was used in the processing while the first [14] was applied as a confirmatory guide to locate the drowning victim in the second. Both videos were sequenced at 25 frames per second. Grey scale images were extracted from the second video [15] starting from the point of initial struggle and cropping was performed to output each image to a 447 by 281 dimension to maintain consistency with the camera coverage to the initial video. Fig. 1. shows a sample image extracted from [14] and another sample image extracted from [15] followed by grey scaling and cropping.

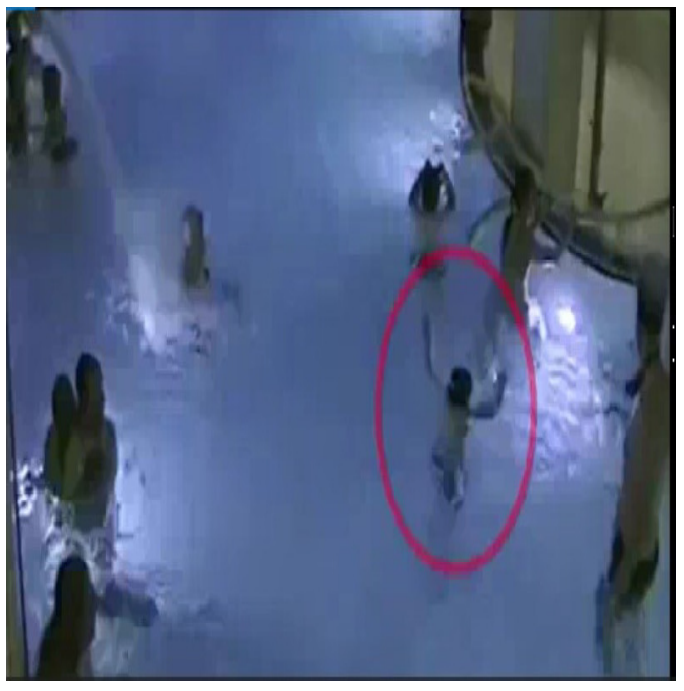

(a)

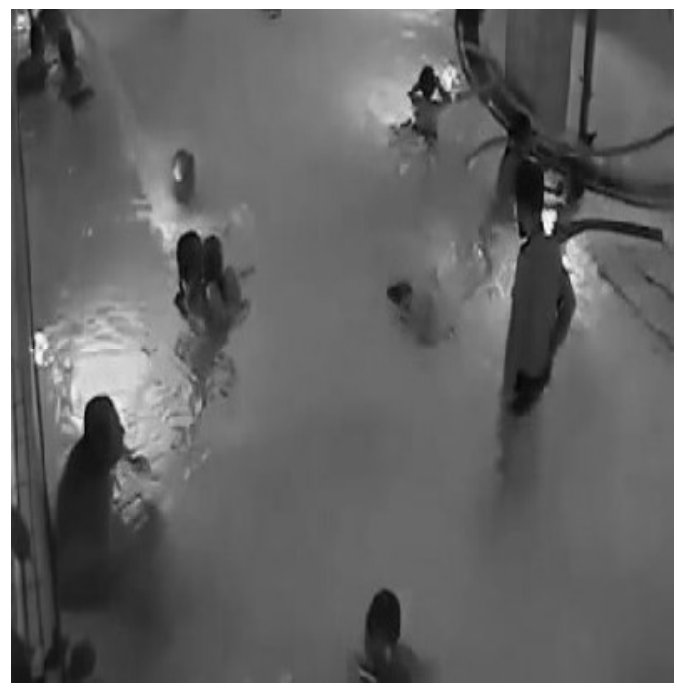

(b)

Fig. 1. (a) Sample image extracted from [14] (b) Sample image extracted from [15] which had been grey scaled and cropped to follow similar camera coverage of the video from [14] 


\subsection{Pre-processing Pipeline}

NEPTUNE's pre-processing pipeline consists of a combination of statistical image processing and K-means clustering [16].

The steps to process the images for every $\mathrm{m}$ seconds of video sequence are summarized below. The steps were repeated for five different values for $\mathrm{m}$ which are 1, 2, 3, 4 and 5 .

1. Grey scaling (cropping was performed for the dataset used in this paper but would not be required in an actual setting)

2. Assuming 5 seconds of video sequences were being processed, for every pixel, the maximum absolute of the Fast Fourier Transform [17] across the 125 images was computed to give a two-dimensional matrix of 447 by 281 . Since the video was sequenced at 25 frames per second, every 5 seconds would have a total 125 images that can be extracted. Similarly, every 4, 3, 2, and 1 seconds of video sequences would be using 100, 75, 50 and 25 images respectively.

3. The values across the two-dimensional matrix were normalized to a range between 0 and 1 to produce another two-dimensional matrix, $\mathrm{N}$.

4. N would be transformed to a 1-dimensional array, 1-N via repeated looping across the $\mathrm{x}$ dimension shadowed by an inner loop across the y-dimension. For instance, the values at position $(1,1),(1,2) \ldots(1,447)$ of $\mathrm{N}$ would be placed at (1), (2) ... (447) of 1-N respectively while the values at position $(2,1),(2,2) \ldots(2,447)$ of $\mathrm{N}$ would be placed at (448), (449) ... (894) respectively.

5. 3-means and 4-means clustering were performed independently on 1-N. An earlier attempt of using 2-means and 3-means clustering resulted in the inability in finding segments either intersecting or close by the struggling victim for some positive video sequences. More segments were created using a 3-means and 4-means clustering and hence increased the probability of finding a segment that either intersects or is very close by the struggling victim for every positive video sequence. The purpose of having two types of clustering was to find pairs of nearest segments, one from $\{\mathrm{Sa}\}$ and another from $\{\mathrm{Sb}\}$ to have a larger pool of variables to explore.

a. From the 3-means clustering, the largest of the 3 clusters was excluded using an assumption that it predominantly consisted of water. The 2 smaller clusters were remapped from 1-N to $\mathrm{N}$ and a set of connected segments $\{\mathrm{Sa}\}$ was extracted. A connected segment is a set of pixels belonging to the same cluster and each pixel within that segment had to be beside any one of the pixels in that segment.

b. From the 4-means clustering, the largest of the 4 clusters was excluded using an assumption that it predominantly consisted of water. The 3 smaller clusters were remapped from $1-\mathrm{N}$ to $\mathrm{N}$ and a second set of connected segments $\{\mathrm{Sb}\}$ was extracted. 
Fig. 2. shows an example of how 3-means clusters would look after remapping 1-N back to N. In comparison to Fig. 1., the cluster with the majority of pixels belonged to water for Fig. 2a. Fig. 2b. illustrates an example of a connected segment.
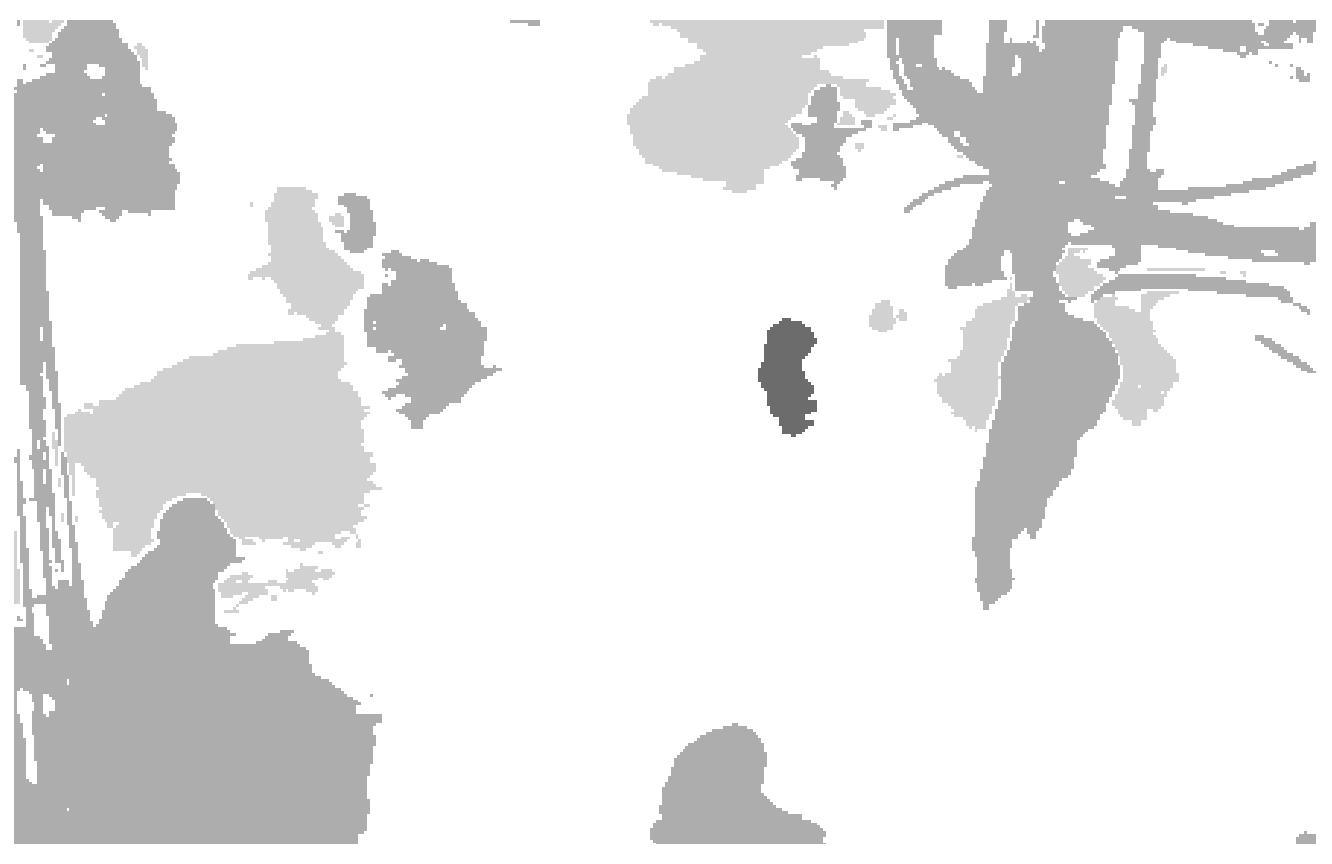

(a)

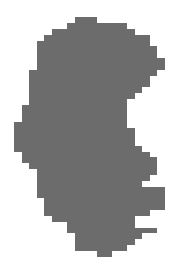

(b)

Fig. 2. (a) 3 clusters created from 3-mean clustering. Water is predominantly within the largest cluster which is shaded in white. The two shades of grey represent the remaining two clusters. The connected segment coloured in black intersects with the struggling victim and belongs to the cluster with the darker shade of grey. (b) A zoomed in image of the single connected segment coloured in black.

6. For each segment in $\{\mathrm{Sa}\}$, variables shown in Table 1 were derived. Variable V1 was reserved for the labelling of presence/absence of a pre-drowning struggling victim within/close by a segment. If there was no segment that intersects the struggling victim, the nearest segment would be considered to contain the pre-drowning struggling victim. 
Table 1. Variables derived for each segment in $\{\mathrm{Sa}\}$

\begin{tabular}{|cc|}
\hline Variable Name & $\begin{array}{c}\text { Variable Description } \\
\text { Ratio of V4 to V3 }\end{array}$ \\
\hline V3 & Number of pixels in the segment \\
\hline V4 & $\begin{array}{r}\text { Standard deviation of values from } 5 \text { points (4 extreme points* and the } \\
\text { segment's centre) }\end{array}$ \\
\hline V5 & Ratio of V2 to the sum of V2 across all segments \\
\hline V6 & Ratio of V3 to the sum of V3 across all segments \\
\hline V7 & Ratio of V4 to the sum of V4 across all segments \\
\hline
\end{tabular}

*4 extreme points are $\{\operatorname{minimum}(\mathrm{x}), \operatorname{minimum}(\mathrm{y})\},\{\operatorname{minimum}(\mathrm{x}), \max (\mathrm{y})\},\{\operatorname{maximum}(\mathrm{x})$, $\operatorname{minimum}(\mathrm{y})\}$ and $\{\operatorname{maximum}(\mathrm{x}), \operatorname{maximum}(\mathrm{y})\}$ where $\mathrm{x}$ and $\mathrm{y}$ are the $\mathrm{x}$-coordinates and $\mathrm{y}$ coordinates of a segment respectively

7. For each segment in $\{\mathrm{Sa}\}$, similar variables for the respective nearest segment from $\{\mathrm{Sb}\}$ was computed as shown in Table 2.

Table 2. Variables derived from respective nearest segment in $\{\mathrm{Sb}\}$

\begin{tabular}{|cc|}
\hline $\begin{array}{c}\text { Variable Name } \\
\text { V8 }\end{array}$ & $\begin{array}{c}\text { Variable Description } \\
\text { Ratio of V10 to V9 }\end{array}$ \\
\hline V9 & Number of pixels in the segment \\
\hline V10 & $\begin{array}{c}\text { Standard deviation of values of } 5 \text { points (4 extreme points* and the } \\
\text { segment's centre) }\end{array}$ \\
\hline V11 & Ratio of V11 to the sum of V11 across all segments \\
\hline V12 & Ratio of V12 to the sum of V12 across all segments \\
\hline V13 & Ratio of V13 to the sum of V13 across all segments \\
\hline
\end{tabular}

*4 extreme points are $\{\operatorname{minimum}(\mathrm{x}), \operatorname{minimum}(\mathrm{y})\},\{\operatorname{minimum}(\mathrm{x}), \operatorname{maximum}(\mathrm{y})\},\{\operatorname{maximum}(\mathrm{x})$, $\operatorname{minimum}(\mathrm{y})\}$ and $\{\operatorname{maximum}(\mathrm{x}), \operatorname{maximum}(\mathrm{y})\}$ where $\mathrm{x}$ and $\mathrm{y}$ are the $\mathrm{x}$-coordinates and $\mathrm{y}$ coordinates of a segment respectively

8. Next, for each segment in $\{\mathrm{Sa}\}$, new variables were created as shown in Table 3 . These new variables were computed using the variables derived from Table 1 and Table 2.

Table 3. Variables derived from respective nearest segment in $\{\mathrm{Sb}\}$

\begin{tabular}{|cc|}
\hline Variable Name & Variable Description \\
\hline V2_8 & Ratio of V2 to V8 \\
\hline V3_9 & Ratio of V3 to V9 \\
\hline V4_10 & Ratio of V4 to V10 \\
\hline V5_11 & Ratio of V5 to V11 \\
\hline V6_12 & Ratio of V6 to V12 \\
\hline V7_13 & Ratio of V7 to V13 \\
\hline
\end{tabular}

9. Finally, the percentile cut-offs for every variable across all segments in $\{\mathrm{Sa}\}$ were computed as shown in Tables A1 (a), (b), (c) and (d) of the Appendix. 
Every variable value for each segment would be assigned either a value of 1,2, 3 or 4 according to the range which they fall into as indicated in Table 4 with respect to length of video sequence. For example, say for a segment in a $5 \mathrm{~s}$ video sequence having a V2 value of 0.03 , it would be transformed to a value of 2 since it is between the 25 th and 50th percentiles of $\mathrm{V} 2$.

Table 4. Value assigned each variable in every segment in $\{\mathrm{Sa}\}$

\begin{tabular}{|cl|}
\hline $\mathbf{1}$ & \multicolumn{1}{c|}{ Range } \\
\hline $\mathbf{2}$ & $\begin{array}{l}\text { being less than or equal to the } 25 \text { th percentile } \\
\text { peing more than the } 25 \text { th percentile but less than or equal to the } 50 \text { th }\end{array}$ \\
\hline $\mathbf{3}$ & $\begin{array}{l}\text { being more than the } 50 \text { th percentile but less than or equal to the } 75 \text { th } \\
\text { percentile }\end{array}$ \\
\hline $\mathbf{4}$ & being more than the 75 th percentile \\
\hline
\end{tabular}

Solely for this training, labelling of V1 for every segment had to be performed. It would contain one of two values; either 1 if it was positive or 0 otherwise. A positive refers to the presence of a struggling pre-drowning victim within or close by the segment. This labelling would not be required for the actual application of NEPTUNE. The video sequence lengths of $1 \mathrm{~s}, 2 \mathrm{~s}, 3 \mathrm{~s}, 4 \mathrm{~s}$ and $5 \mathrm{~s}$ respectively contained $36,17,12,9$, and 8 positive video sequences. A positive video sequence would each entail a struggling pre-drowning victim. Correspondingly, for the video sequence lengths of $1 \mathrm{~s}, 2 \mathrm{~s}, 3 \mathrm{~s}, 4 \mathrm{~s}$ and $5 \mathrm{~s}$, the respective number of video sequences for which there were no struggling were 946, 406, 269, 179 and 138. Each video sequence may have multiple segments with at most 1 positive segment.

\subsection{Equations Derivation}

The equations were derived from optimized rules generated via association rules mining [18]. Association rules mining was attempted as an approach to detect the positives as anomalies. The existence of a non-linear relationship between the variables and the presence/absence of positives is shown in Fig. 3 where there is a poor correlation not exceeding 0.37 between the actual and predicted scores for all the video sequence lengths studied. This further justifies the use of association rules mining since it can identify both linear and non-linear properties that would distinguish positives from non-positives. The formulae of the regression model used in Fig. 3a, b, $\mathrm{c}, \mathrm{d}$ and e are shown in Table A2 of the Appendix. 


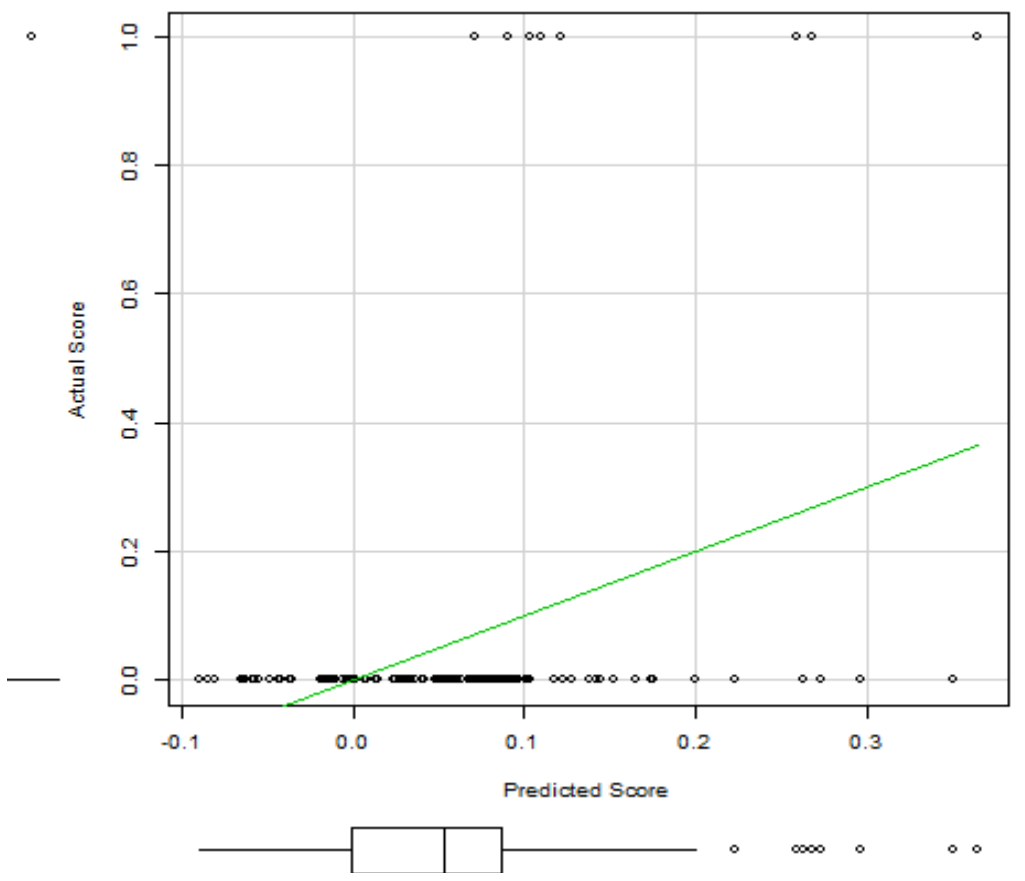

(a) Video Sequence Length of $5 \mathrm{~s}$

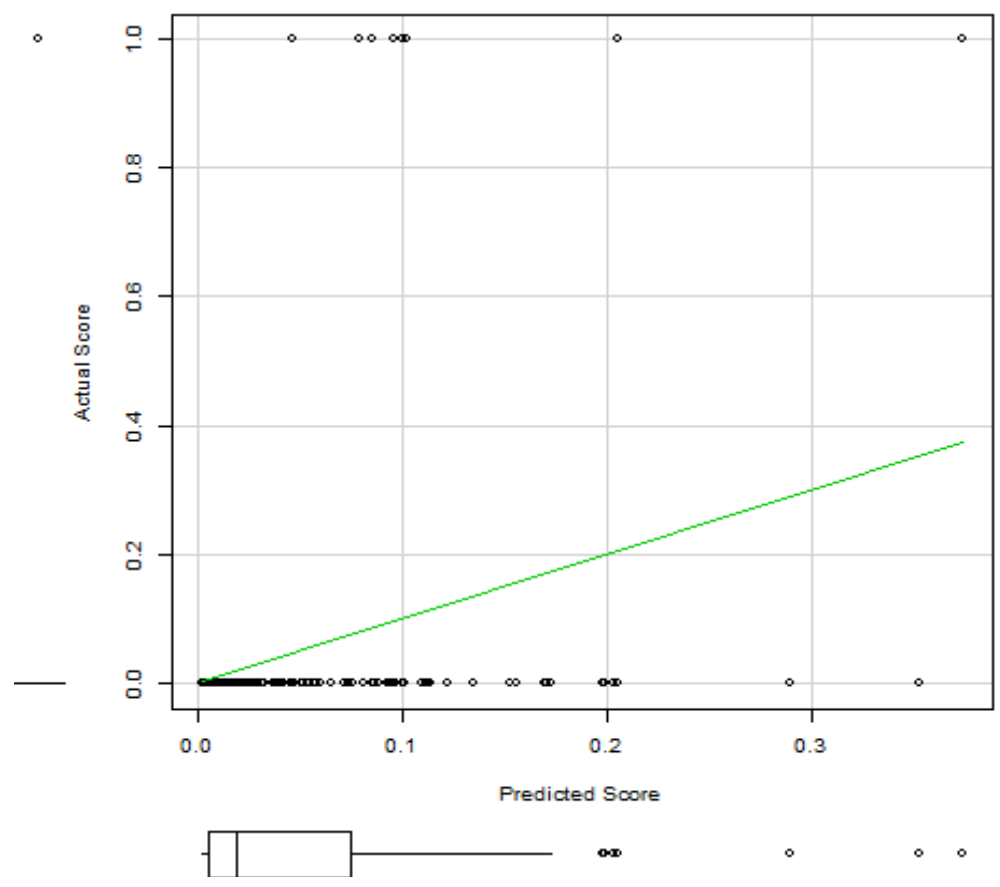

(b) Video Sequence Length of $4 \mathrm{~s}$ 


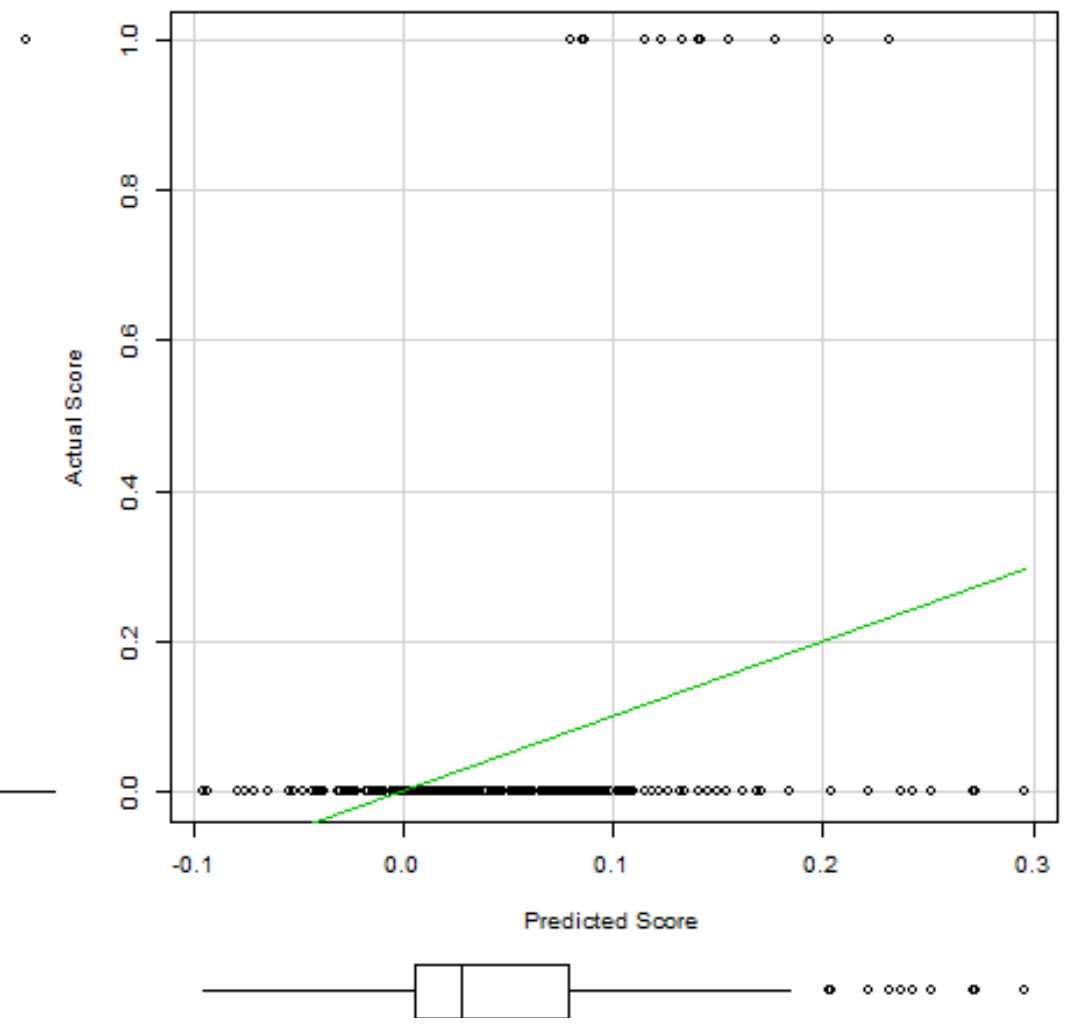

(c) Video Sequence Length of 3s

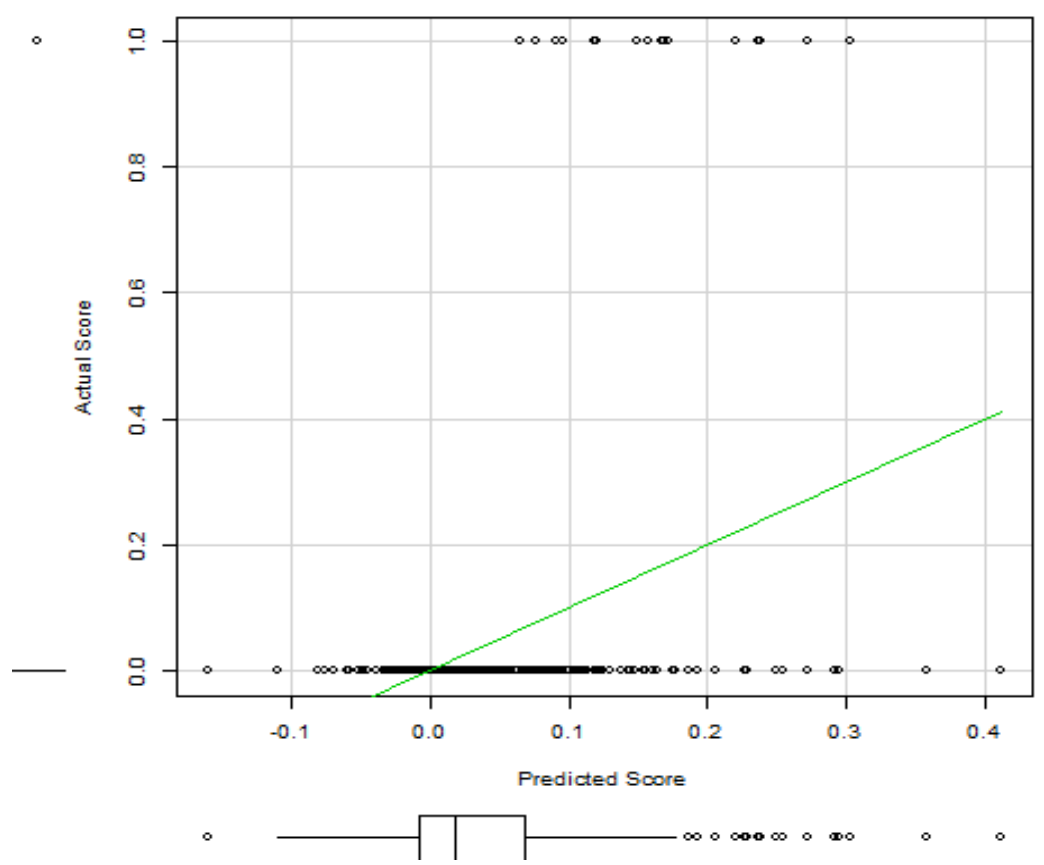

(d) Video Sequence Length of $2 \mathrm{~s}$ 


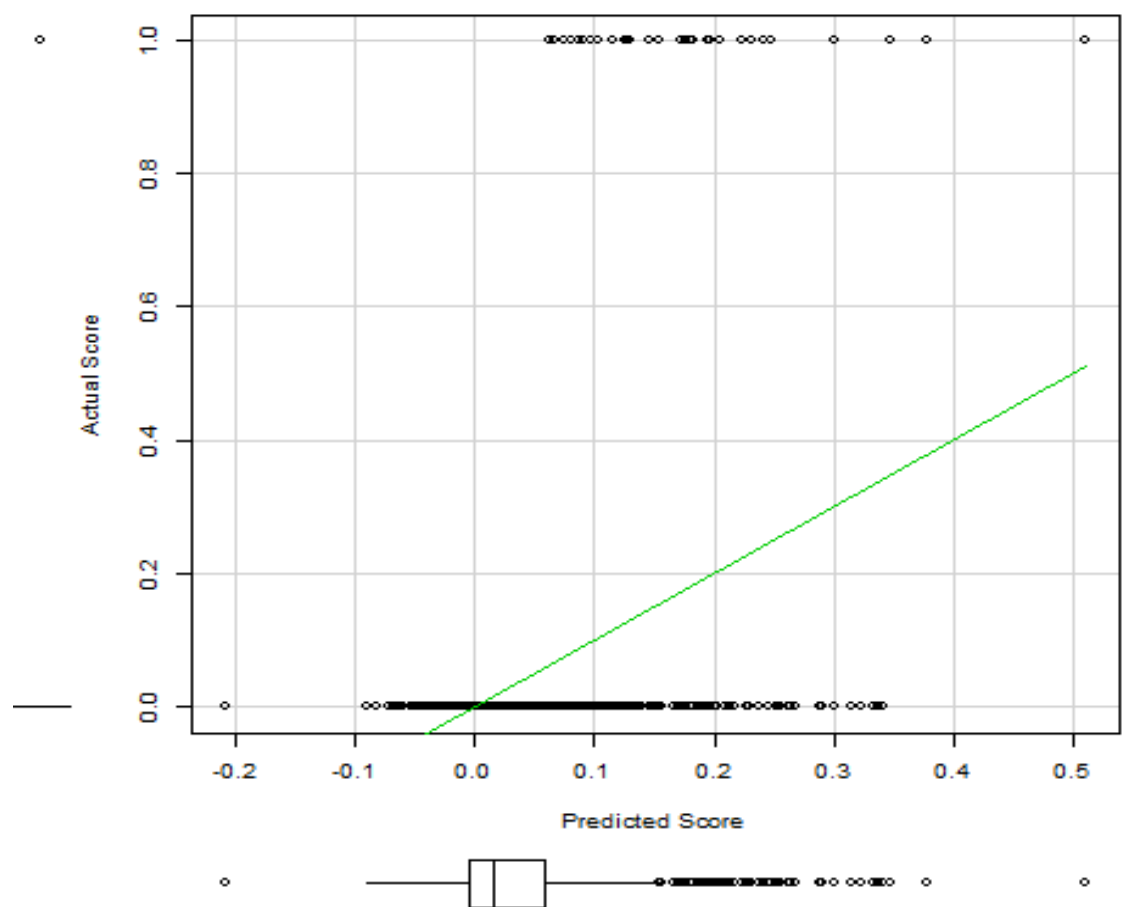

(e) Video Sequence Length of $1 \mathrm{~s}$

Fig. 3. The predicted scores created via a AIC stepwise linear regression model [19] built using the entire dataset against the actual scores for the various video sequence lengths. A score of 1 denotes a positive.

For video sequence lengths of $4 \mathrm{~s}$ and $5 \mathrm{~s}$, the positive segments with the highest predicted score had the largest and most circular segment as shown in Fig. 4 and 5 which made these two positive segments more distinguishable than the other positive segments within the respective video sequence lengths.

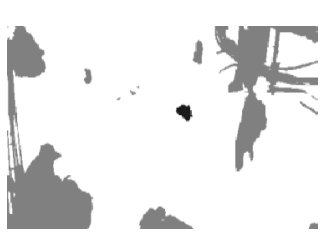

(a) $1 \mathrm{st} 5$ seconds

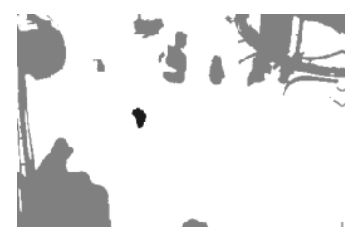

(b) 2nd 5 seconds

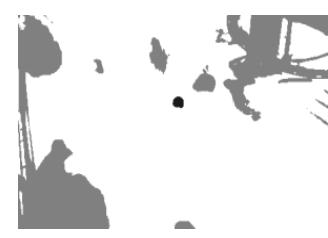

(c) 3rd 5 seconds

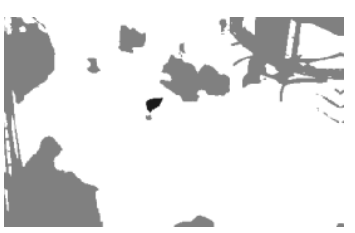

(d) 4th 5 seconds

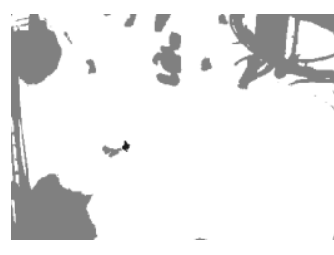

(e) 5 th 5 seconds

(f) 6th 5 seconds

(g) 7th 5 seconds

(h) 8th 5 seconds

Fig. 4. The positive segments shaded in black for the first 8 video sequences of length $5 \mathrm{~s}$ where there was a struggling victim. The 7th 5 seconds video sequence had the highest prediction score using linear regression. 


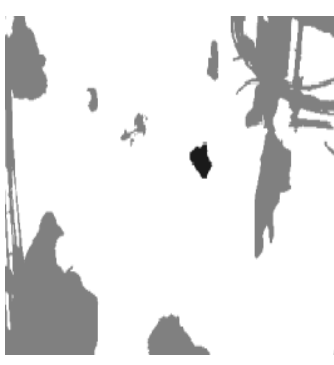

(a) 1 st 4 seconds

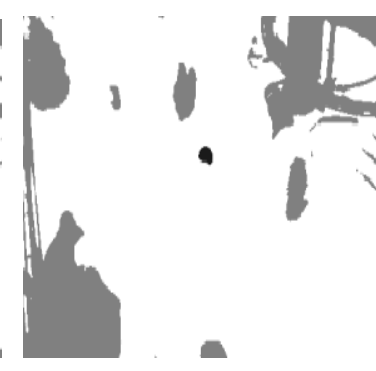

(b) 2nd 4 seconds

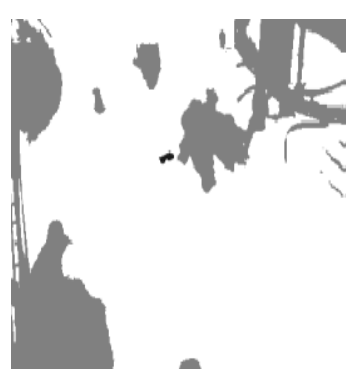

(c) 3rd 4 seconds

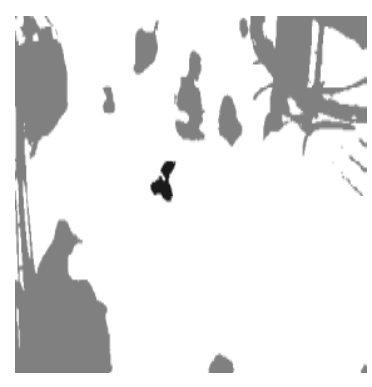

(d) 4 th 4 seconds
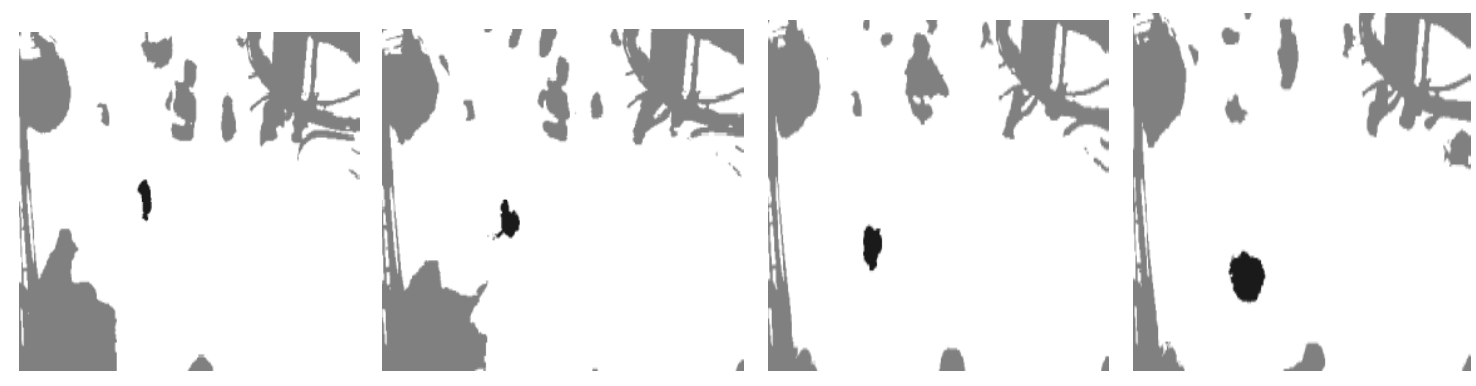

(e) 5 th 4 seconds

(g) 7 th 4 seconds (h) 8th 4 seconds

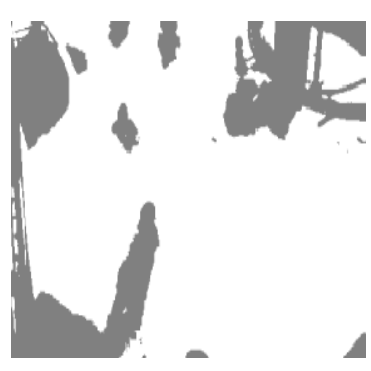

(i) 9th 4 seconds

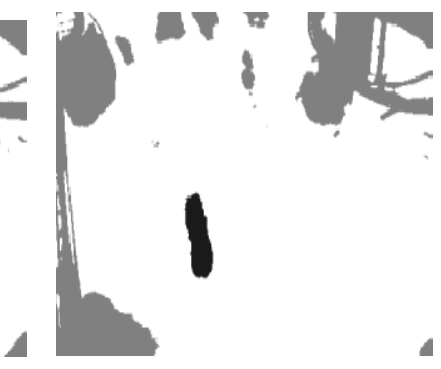

(j) 10th 4 seconds

Fig. 5. The positive segments shaded in black for the first 10 video sequences of length $4 \mathrm{~s}$ where there was a struggling victim. The 8 th 4 seconds video sequence had the highest prediction score using linear regression. No positive segment was detectable in 9 th 4 seconds video sequence although there was a struggling.

Rules were generated independently for the various video sequence lengths to detect presence/absence of a positive segment using 19 variables of which 18 came from Tables 1, 2 and 3. One of the 19 variables, was the label for the absence/presence of a positive. All rules were generated for a confidence of 1 and targeted a minimum of 1 positive. We generated different rule sets across different number of variables ranging from 3 to the full 19. For every number of variables used for rule set generation, one had to be the label. For instance, if 3 variables were used, 2 would be derived variables and 1 would be the label. Fig. 6 . shows that the number of number of positives identifiable for various number of variables used across the various video sequence lengths. 


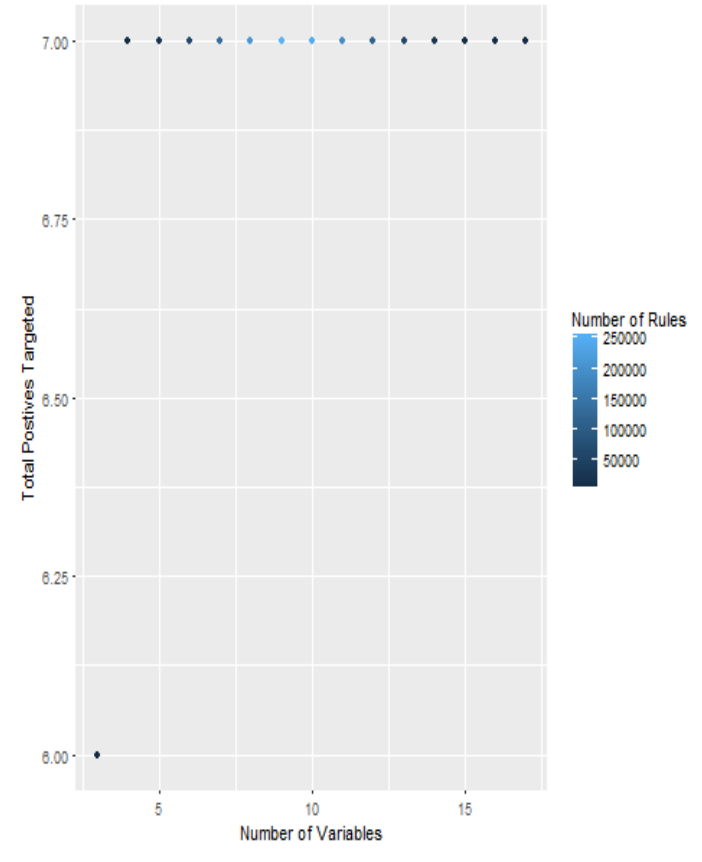

(a) Video Sequence Length of $5 \mathrm{~s}$

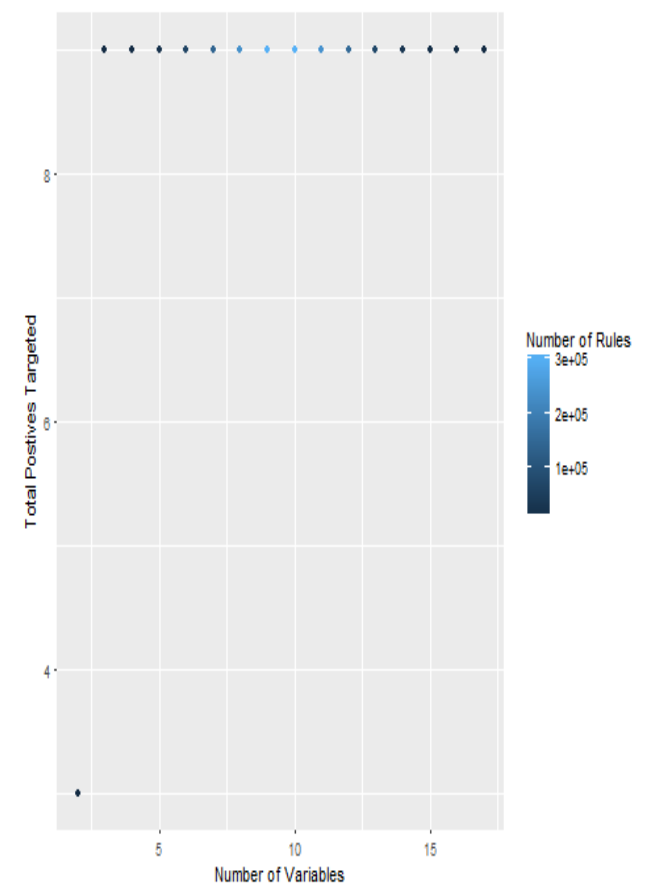

(c) Video Sequence Length of $3 \mathrm{~s}$

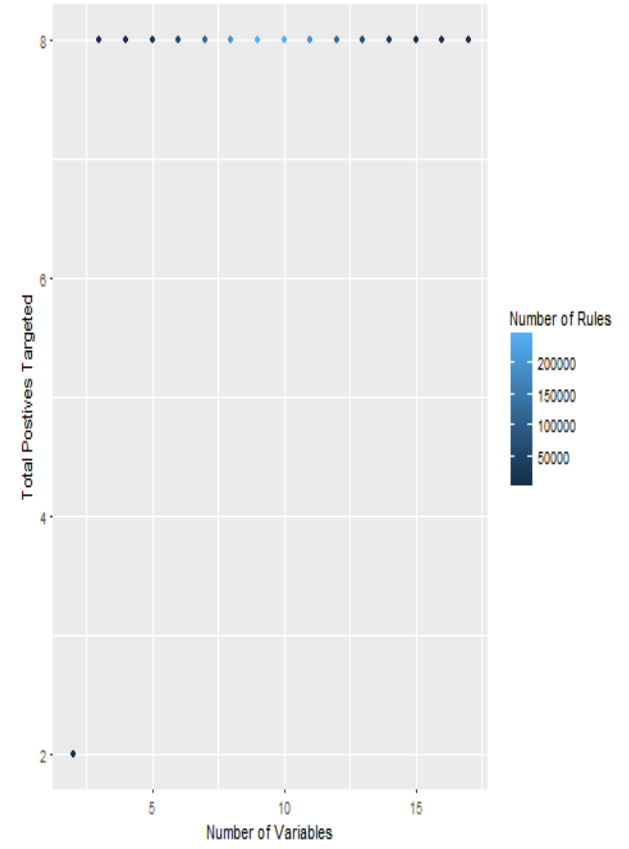

(b) Video Sequence Length of $4 \mathrm{~s}$

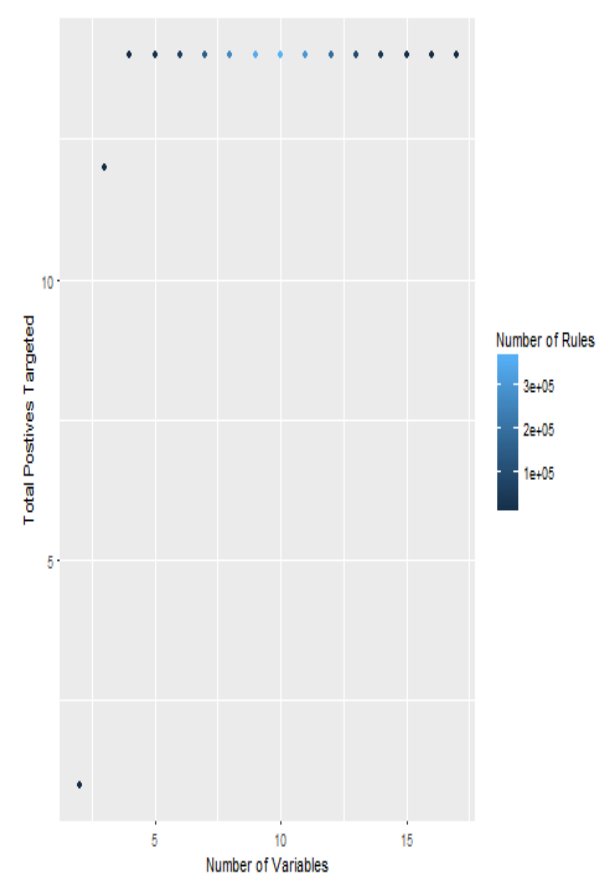

(d) Video Sequence Length of $2 \mathrm{~s}$ 


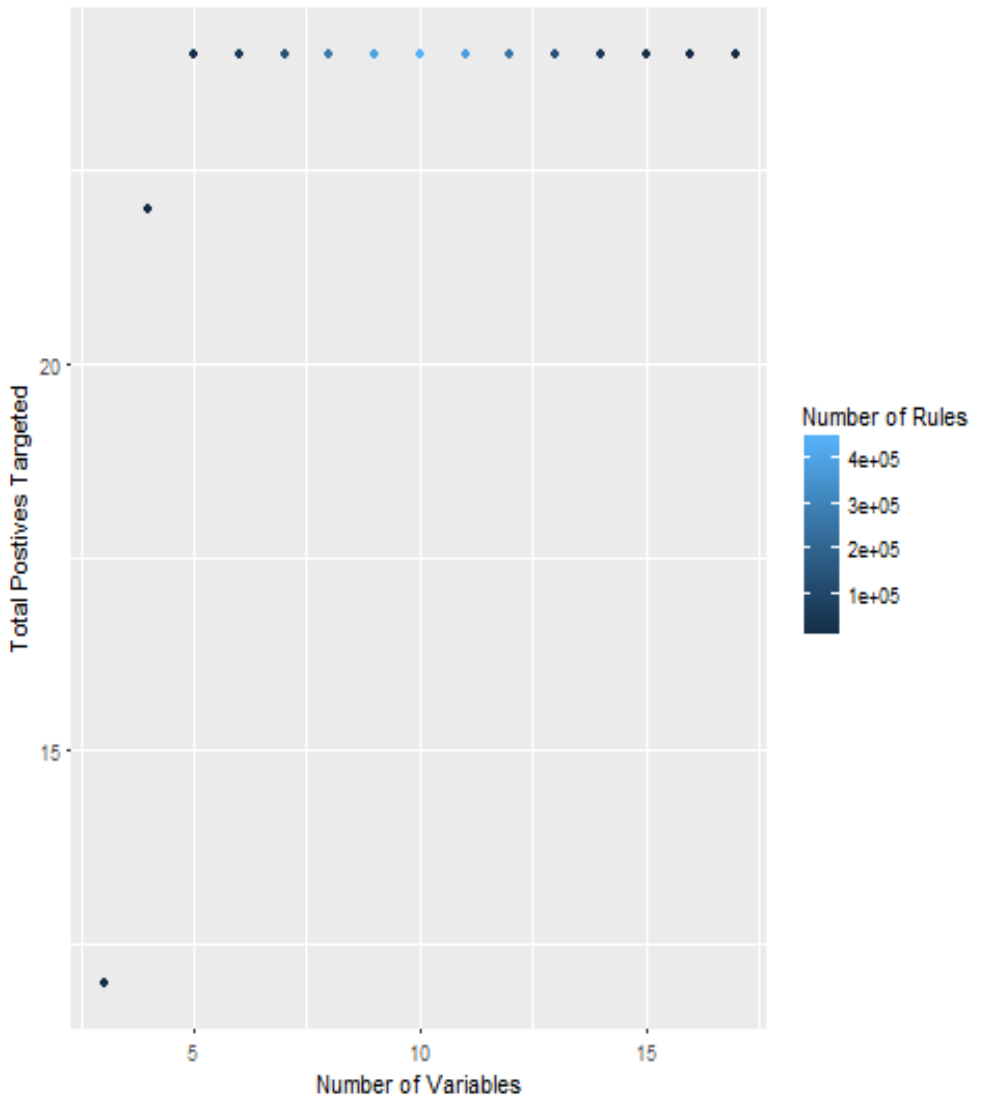

(e) Video Sequence Length of 1s

Fig. 6. Total positives targeted and number of rules generated with $100 \%$ confidence in detecting positives across the corresponding number of variables used in the rules generation. Each spot represents a rule set consisting of rules generated for the respective number of variables. Rule sets which targeted the maximum number of positives detectable for the respective video sequences lengths were enclosed within a red bounding box

The proportion of the rules generated using variables that detected the maximum number of positives either targeting 2 or 3 positives across the different video sequence lengths are shown in Fig. 7. Having a higher proportion of individual rules targeting more positives would ensure that the rule set is more generic and hence applicable across other datasets. Therefore, for the final rule set in each of the video sequence lengths studied, rules generated with the highest proportion of individual rules targeting the most number of positives would be chosen. It can be seen in Fig. 7. that the highest proportion rules targeting 2 or 3 positives tend to be generated when less variables were. Using less variables would reduce the specificity of the rules generated thereby allowing more generic rules to be produced. Each rule in final rule set would be henceforth referred to as an equation. 


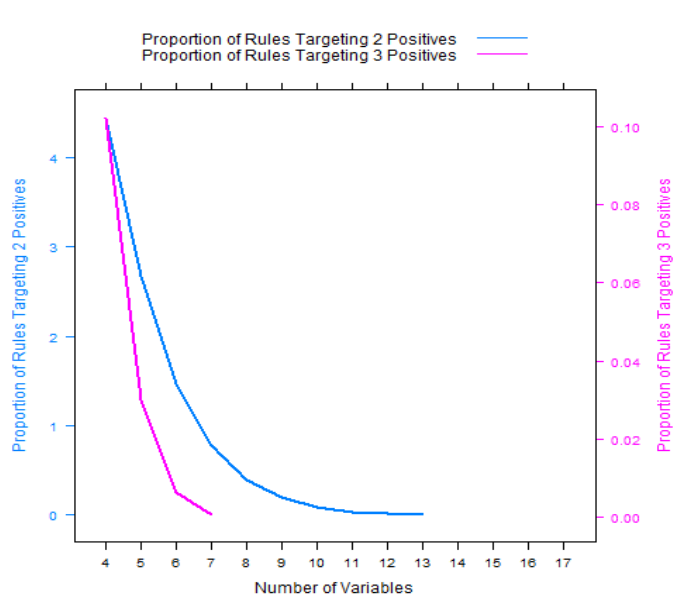

(a) Video Sequence Length of $5 \mathrm{~s}$

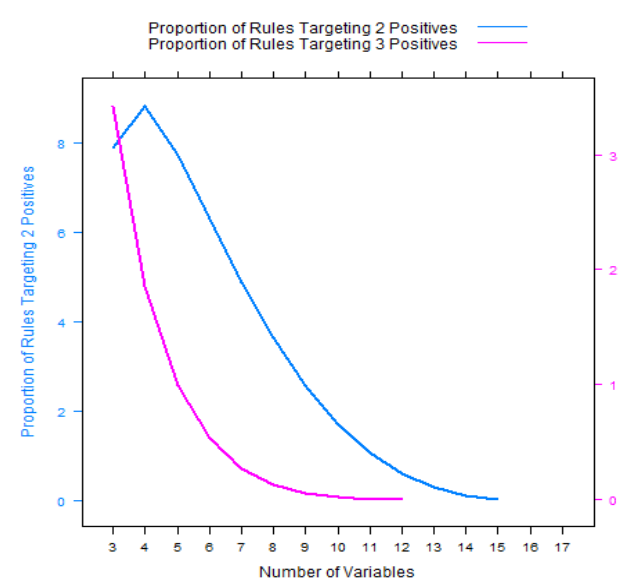

(c) Video Sequence Length of $3 \mathrm{~s}$

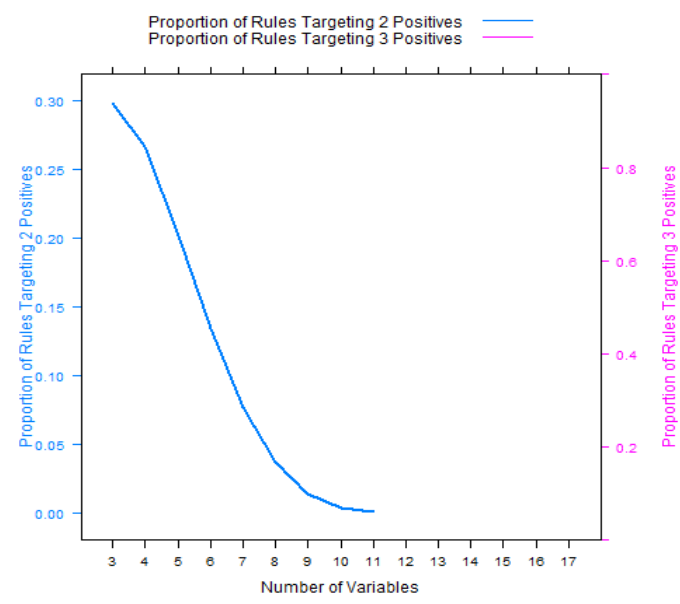

(b) Video Sequence Length of $4 \mathrm{~s}$

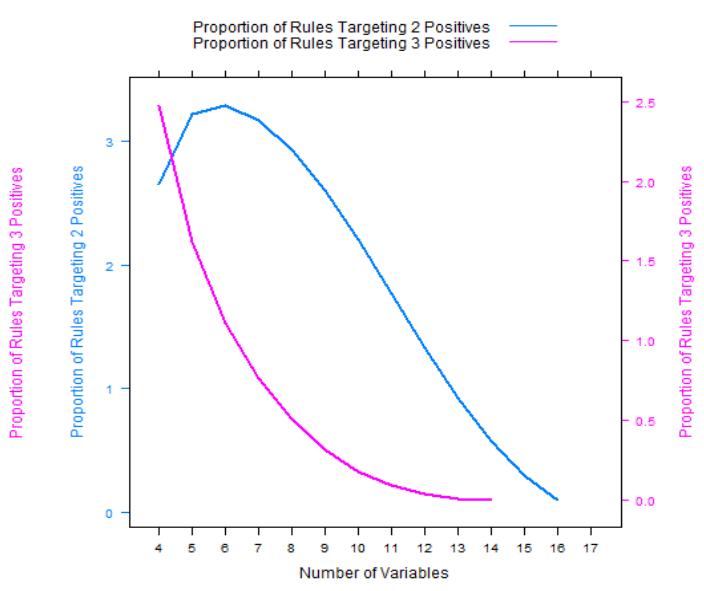

(d) Video Sequence Length of $2 \mathrm{~s}$

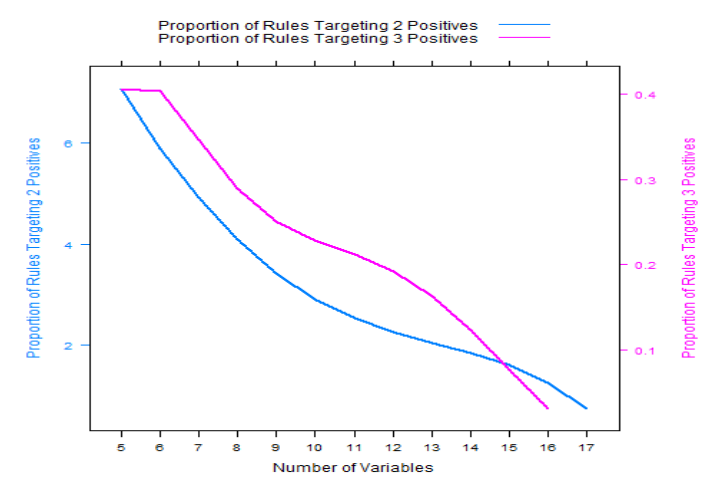

(e) Video Sequence Length of $1 \mathrm{~s}$

Fig. 7. Proportion of rules targeting 2 or 3 positives across the various number of variables used for rules generation 


\section{RESULTS}

Fig. 8. shows the positive detections during the 40s of struggle. It should be noted there were time points when only one of the video sequence lengths detected the presence of a struggle. For instance, at the 39th second of struggle, only the video sequence length of $3 \mathrm{~s}$ could detect the struggle. Hence, it is proposed to use all the video sequence lengths in parallel for pre-drowning struggling detection to maximum the detectable time points of struggle.

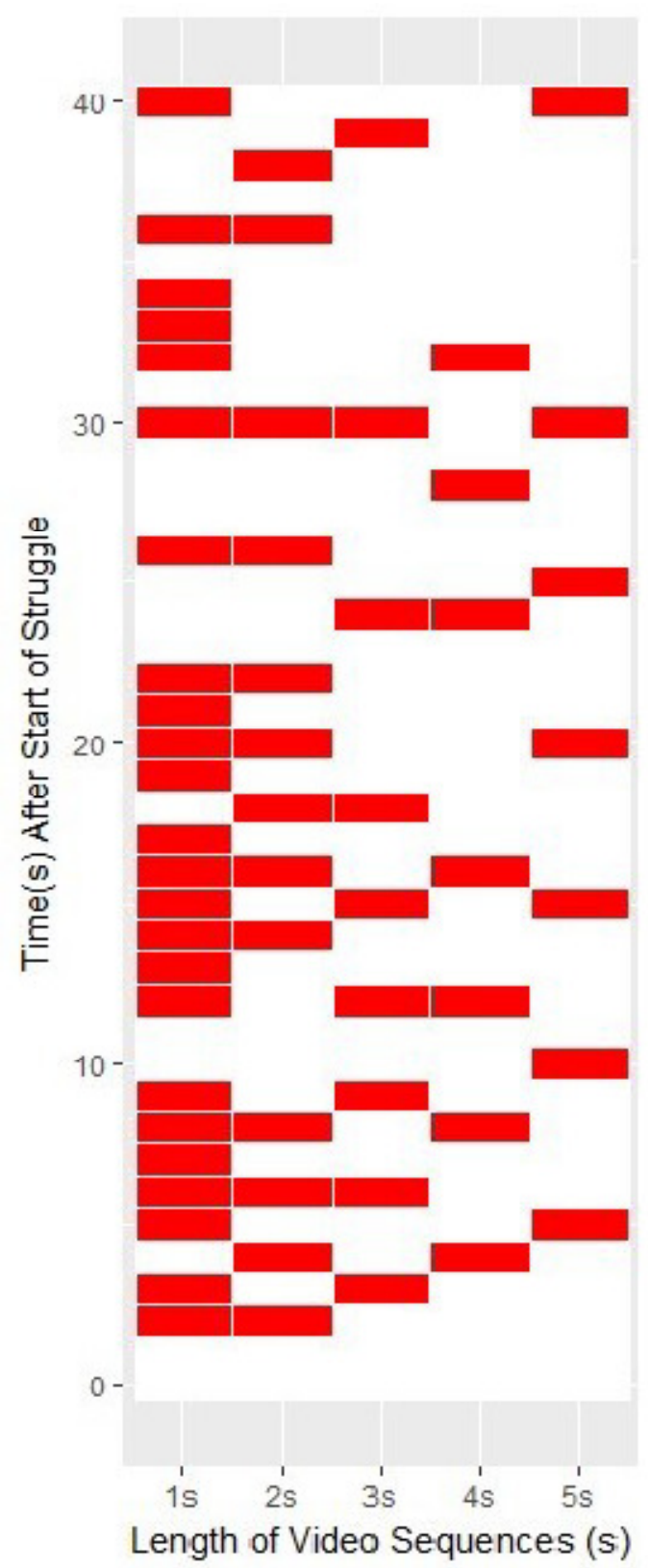

Fig. 8. Detection during the $40 \mathrm{~s}$ of struggle for the various video sequence lengths. Positive detection only occurs at the time points where there is a red rectangle. 


\section{CONCLUSION}

NEPTUNE was presented in this paper as a feasible technique for early detection of struggling pre-drowning victims. The reliance on solely camera based video sequences would allow an easy integration into existing infrastructure in swimming pools with camera(s). This is the first vision based technique built using real video sequences and the fastest requiring at least 1 but not more than 5 seconds of video footage for detection. With collaborative initiatives to collate more actual pre-drowning video footages, NEPTUNE can be refined and further developed into a real-time cost-effective vision based early pre-drowning detection system.

\section{Acknowledgments}

This work has been supported by IAG Firemarks Singapore. I like to thank everyone in the IAG Firemarks Singapore team for their continued encouragement especially Allison Howells for her initial and continued cheering of what started as a simple possibility to a prospective reality.

\section{REFERENCES}

[1] World Health Organization (WHO), Drowning Fact Sheet (2018), http://www.who.int/mediacentre/factsheets/fs347/en/

[2] United States Consumer Product Safety Commission, Fatal Child Pool and Spa Drownings (2017), https://www.cpsc.gov/Latest-Pool-Safely-Stats-At-Least-163-Children-Fatally-Drowned-in-Poolsand-Spas-This-Summer

[3] Lasbeur L, Szego-Zguem E, Guillam M, et al. "671 Epidemiological surveillance of drowning: a national survey in France, 1 June to 30 September 2015". Injury Prevention 22 (2016): A241.

[4] Bierens, Joost, and Andrea Scapigliati. "Drowning in swimming pools." Microchemical journal 113 (2014): 53-58.

[5] Zhang, Chi, Xiaoguang Li, and Fei Lei. "A Novel Camera-Based Drowning Detection Algorithm." Advances in Image and Graphics Technologies, Springer Berlin Heidelberg (2015): 224-233.

[6] Fei, Lei, Wang Xueli, and Chen Dongsheng. "Drowning Detection Based on Background Subtraction." Proceedings of the Sixth IEEE International Conference on Embedded Software and Systems (ICESS 2009).

[7] How-Lung Eng, Kar-Ann Toh, Wei-Yun Yau, Junxian Wang. "DEWS: A Live Visual Surveillance System for Early Drowning Detection at Pool." IEEE transactions on circuits and systems for video technology 18:2 (2008): 194-210.

[8] Wenmiao Lu,Yap-Peng Tan, Yap-Peng Tan. "A Vision-Based Approach to Early Detection of Drowning Incidents in Swimming Pools." IEEE Transactions on Circuits and Systems for Video Technology 14:2 (2004):159 - 178 .

[9] How-Lung Eng, Kar-Ann Toh, Alvin H. Kam, Junxian Wang and Wei-Yun Yau. "An automatic drowning detection surveillance system for challenging outdoor pool environments". Proceedings of the Ninth IEEE International Conference on Computer Vision (ICCV 2003). 
[10] Nasrin Salehi, Maryam Keyvanara, Seyed Amirhassan Monadjemmi. "An Automatic Video-based Drowning Detection System for Swimming Pools Using Active Contours." I.J. Image, Graphics and Signal Processing 8:8 (2016): 1-8.

[11] S. Nagalikitha, A.V. Kiranmai. "Automatic Waist Airbag Drowning Prevention System Based on Motion Information Measured by Memos Accelerometer and pressure." International Journal of Emerging Trends in Engineering Research (IJETER) 3:6 (2015): 204-206.

[12] Mohamed Kharrat, Yuki Wakuda, Shinsuke Kobayashi, Noboru Koshizuka, Ken Sakamura. "Near drowning detection system based on swimmer's physiological information analysis." Proceedings of the Third World Conference on Drowning Prevention (2011).

[13] McAdams, E., Krupaviciute, A., Gehin, C., Grenier, E., Massot, B., Dittmar, A., Rubel, P., Fayn, J."Wearable sensor systems: The challenges.", In Proceedings of the 33rd Annual International Conference of the IEEE Engineering in Medicine Biology Society (EBMC 2011): 3648-3651.

[14] Mirror, World News (2017). https://www.mirror.co.uk/news/world-news/horror-five-year-old-boy-10585736

[15] You Tube (2017). "A 5-year-old child drowns in a swimming pool without anyone noticing (Finland)"https://www.youtube.com/watch?v=zuZIfy4aBEY

[16] Hartigan, J. A. and Wong, M. A. "Algorithm AS 136: A K-means clustering algorithm." Applied Statistics 28 (1979): 100-108

[17] Gentleman, W. M. and Sande, G. "Fast Fourier Transforms: For Fun and Profit". In Proceedings of the November 7-10, 1966, Fall Joint Computer Conference (AFIPS '66 (Fall)): 563-578.

[18] Michael H., Bettina G. and Kurt H. "arules - A Computational Environment for Mining Association Rules and Frequent Item Sets."Journal of Statistical Software 14:15 (2005):1-25.

[19] Hastie, T. J. and Pregibon, D. "Generalized linear models. Chapter 6 of Statistical Models in S"(1992). 


\section{Appendix}

Table A1 (a). Percentile cut-offs for each variable across all segments in $\left\{\mathbf{S}_{\mathbf{a}}\right\}$ using 5 s of video sequences

\begin{tabular}{c|c|c|c}
\hline Variable Name & 25th Percentile & 50th Percentile & 75th Percentile \\
\hline V2 & 0.025537 & 0.089672 & 0.136088 \\
\hline V3 & 4 & 14.5 & 232.5 \\
\hline V4 & 0.616663 & 1.83083 & 5.959598 \\
\hline V5 & 0.033195 & 0.079342 & 0.160908 \\
\hline V6 & 0.002247 & 0.012159 & 0.144687 \\
\hline V7 & 0.017574 & 0.054455 & 0.176347 \\
\hline V8 & 0.03157 & 0.095445 & 0.197203 \\
\hline V9 & 5 & 23 & 187.75 \\
\hline V10 & 0.817409 & 2.087051 & 6.102764 \\
\hline V11 & 0.001802 & 0.005235 & 0.012307 \\
\hline V12 & 0.000165 & 0.000753 & 0.006355 \\
\hline V13 & 0.002531 & 0.005998 & 0.015984 \\
\hline V2_8 & 0.543143 & 0.808972 & 1.39471 \\
\hline V3_9 & 0.38125 & 1.294801 & 2.176598 \\
\hline V4_10 & 0.475384 & 1.027277 & 1.501146 \\
\hline V5_11 & 7.80108 & 14.34464 & 29.0312 \\
\hline V6_12 & 5.501654 & 15.77497 & 50.7646 \\
\hline V7_13 & 4.08387 & 9.045111 & 18.00813 \\
\hline & & & \\
\hline
\end{tabular}

Table A1 (b). Percentile cut-offs for each variable across all segments in $\left\{\mathbf{S}_{\mathbf{a}}\right\}$ using 4 s of video sequences

\begin{tabular}{c|c|c|c}
\hline Variable Name & 25th Percentile & 50th Percentile & 75th Percentile \\
\hline V2 & 0.028521 & 0.096291 & 0.181219 \\
\hline V3 & 4 & 13 & 175.5 \\
\hline V4 & 0.552183 & 1.605571 & 5.200332 \\
\hline V5 & 0.036251 & 0.094939 & 0.158282 \\
\hline V6 & 0.002502 & 0.011834 & 0.130142 \\
\hline V7 & 0.017525 & 0.055982 & 0.15279 \\
\hline V8 & 0.028882 & 0.092357 & 0.197203 \\
\hline V9 & 4 & 14 & 258.5 \\
\hline V10 & 0.555836 & 1.951793 & 6.336658 \\
\hline V11 & 0.001904 & 0.006055 & 0.013018 \\
\hline V12 & 0.000128 & 0.000523 & 0.008301 \\
\hline V13 & 0.001747 & 0.00508 & 0.017826 \\
\hline V2_8 & 0.645632 & 0.89624 & 1.350195 \\
\hline V3_9 & 0.5 & 1.12129 & 2.333333 \\
\hline V4_10 & 0.560464 & 1.005624 & 1.50856 \\
\hline V5_11 & 6.285207 & 12.07338 & 27.94023 \\
\hline V6_12 & 7.657875 & 15.97658 & 56.00498 \\
\hline V7_13 & 5.056071 & 9.226469 & 21.33621 \\
\hline
\end{tabular}


Table A1 (c). Percentile cut-offs for each variable across all segments in $\left\{\mathbf{S}_{\mathbf{a}}\right\}$ using 3 s of video sequences

\begin{tabular}{c|c|c|c}
\hline Variable Name & 25th Percentile & 50th Percentile & 75th Percentile \\
\hline V2 & 0.03194 & 0.096123 & 0.150733 \\
\hline V3 & 4 & 13 & 159 \\
\hline V4 & 0.555836 & 1.566484 & 5.153229 \\
\hline V5 & 0.030702 & 0.082569 & 0.143622 \\
\hline V6 & 0.002227 & 0.01207 & 0.106299 \\
\hline V7 & 0.018207 & 0.047486 & 0.136789 \\
\hline V8 & 0.027022 & 0.096123 & 0.197203 \\
\hline V9 & 3 & 11 & 212 \\
\hline V10 & 0.394405 & 1.457435 & 6.193818 \\
\hline V11 & 0.001662 & 0.005614 & 0.011111 \\
\hline V12 & 0.000108 & 0.000351 & 0.007508 \\
\hline V13 & 0.001214 & 0.003857 & 0.015949 \\
\hline V2_8 & 0.588914 & 0.856317 & 1.418486 \\
\hline V3_9 & 0.5 & 1.402542 & 2.5 \\
\hline V4_10 & 0.706932 & 1.084431 & 1.927521 \\
\hline V5_11 & 6.548546 & 13.00316 & 29.72699 \\
\hline V6_12 & 8.128327 & 19 & 64.53311 \\
\hline V7_13 & 5.06461 & 10.99971 & 23.4315 \\
\hline
\end{tabular}

Table A1 (d). Percentile cut-offs for each variable across all segments in $\left\{\mathbf{S}_{\mathbf{a}}\right\}$ using $2 \mathrm{~s}$ of video sequences

Variable Name

\begin{tabular}{c|c|c|c} 
Variable Name & 25th Percentile & 50th Percentile & 75th Percentile \\
\hline V2 & 0.027442 & 0.096123 & 0.181219 \\
\hline V3 & 4 & 11 & 185 \\
\hline V4 & 0.544352 & 1.31045 & 5.561496 \\
\hline V5 & 0.032004 & 0.081686 & 0.140064 \\
\hline V6 & 0.001807 & 0.006944 & 0.110295 \\
\hline V7 & 0.015953 & 0.039461 & 0.137062 \\
\hline V8 & 0.028312 & 0.096123 & 0.197203 \\
\hline V9 & 4 & 15 & 232 \\
\hline V10 & 0.555836 & 1.972027 & 6.934729 \\
\hline V11 & 0.001601 & 0.004623 & 0.010108 \\
\hline V12 & 0.000116 & 0.000536 & 0.007775 \\
\hline V13 & 0.00136 & 0.004605 & 0.016561 \\
\hline V2_8 & 0.653128 & 0.897169 & 1.275932 \\
\hline V3_9 & 0.5 & 1.25969 & 2.084053 \\
\hline V4_10 & 0.502151 & 1.019212 & 1.580454 \\
\hline V5_11 & 8.305886 & 13.30195 & 28.31558 \\
\hline V6_12 & 6.32996 & 16.15734 & 48.24208 \\
\hline V7_13 & 4.833126 & 10.26422 & 23.45575 \\
\hline
\end{tabular}


Table A1 (d). Percentile cut-offs for each variable across all segments in $\left\{\mathbf{S}_{\mathbf{a}}\right\}$ using $1 \mathrm{~s}$ of video sequences

\begin{tabular}{c|c|c|c}
\hline Variable Name & 25th Percentile & 50th Percentile & 75th Percentile \\
\hline V2 & 0.043662 & 0.102924 & 0.197203 \\
\hline V3 & 3 & 8 & 109.75 \\
\hline V4 & 0.394405 & 1.099948 & 4.615003 \\
\hline V5 & 0.032882 & 0.074918 & 0.12968 \\
\hline V6 & 0.001575 & 0.006651 & 0.073862 \\
\hline V7 & 0.01319 & 0.034193 & 0.119747 \\
\hline V8 & 0.039327 & 0.111167 & 0.197203 \\
\hline V9 & 3 & 10 & 123 \\
\hline V10 & 0.547526 & 1.344413 & 5.361706 \\
\hline V11 & 0.001796 & 0.005085 & 0.008561 \\
\hline V12 & 0.000106 & 0.000323 & 0.004121 \\
\hline V13 & 0.001244 & 0.003122 & 0.012171 \\
\hline V2_8 & 0.043662 & 0.102924 & 0.197203 \\
\hline V3_9 & 3 & 8 & 109.75 \\
\hline V4_10 & 0.394405 & 1.099948 & 4.615003 \\
\hline V5_11 & 0.032882 & 0.074918 & 0.12968 \\
\hline V6_12 & 0.001575 & 0.006651 & 0.073862 \\
\hline V7_13 & 0.01319 & 0.034193 & 0.119747 \\
\hline
\end{tabular}

Table A2. Regression formulae for the respective models in Fig 3.

\begin{tabular}{|c|c|}
\hline Fig & Formula \\
\hline $3 a$ & $\begin{array}{c}V 1=-0.6882 * V 2+0.8153 * V 6-1.1143 * V 7-0.0003874 * V 9+13.0915 * V 12 \\
-0.001769 * V 6 \_12+0.002818 * V 7 \_13+0.1227\end{array}$ \\
\hline $3 b$ & $V 1=0.01103 * V 4+0.00107 * V 15-0.00123$ \\
\hline $3 c$ & $V 1=-0.3232 * V 2+0.0001431 * V 3-0.1553 * V 6-0.2502 * V 8-0.09636$ \\
\hline $3 d$ & $\begin{array}{c}V 1=0.01665 * V 4-0.1753 * V 6-0.2300 * V 8+0.0002441 * V 9-7.6024 * V 12 \\
-0.005745 * V 4 \_10+0.03580\end{array}$ \\
\hline $3 e$ & $\begin{aligned} & V 1=-0.2072 * V 2+0.01528 * V 4-0.1563 * V 6+0.000243 * V 9-0.01994 * V 10 \\
&-2.9549 * V 11-7.4156 * V 12+7.6710 * V 13-0.004826 * V 16+0.58134\end{aligned}$ \\
\hline
\end{tabular}

